\title{
Analysis of Factors Affecting the Event of Diarrhea in Infants Age 0 to 2 Years
}

\author{
Antika Maulida Rahayu ${ }^{1, *}$, Ismarwati \\ ${ }^{1}$ Student of Masters Department of Midwifery, Faculty of Health Sciences, Yogyakarta 'Aisyiyah University, \\ Indonesia \\ ${ }^{2}$ Lecturer, Department of Midwifery, Faculty of Health Sciences, Yogyakarta 'Aisyiyah University, Indonesia \\ * corresponding author
}

Submission date: 10 Juli 2018, Receipt date: 10 Oktober 2019, Publication date: 1 Juli 2020

\section{Abstract}

Diarrhea is the number 2 cause of death among children under five in the world, number 3 in infants, and number 5 for all ages. There are several factors that influence the occurrence of diarrhea in infants apart from infectious factors (bacterial infections, viral infections, parasitic infections, parenteral infections). Other contributing factors are attainment of breastfeeding, food factors, and water sources used daily. The aim of this literature review is to analyze the factors that influence the incidence of diarrhea in infants aged 0 to 2 years. a process using the search stage to conduct a literature review. A search of relevant databases as well as literature and reference lists of included studies was carried out. An analysis of 10 journals conducted by researchers showed that infants aged 10 months who were fully breastfed when associated with diarrhea had lower scores $(O R=0.59,95 \%$ CI 0.43-0.82), while children -children who received food and candy or milk were associated with an increased prevalence of diarrhea, namely $(O R=1.64,95 \% C I 1.22-2.22$ and $O R=1.67,95 \%$ CI 1.21-2.30). And 2.58 times higher in homes with damaged water filters $(O R=2.58,95 \%$ CI 0.1, 10.1) compared to homes with working water filters. $15 \%$ of families with good water filters reported diarrhea, while 31\% of families with damaged water filters reported diarrhea. Exclusive breastfeeding for up to 6 months can reduce the risk of diarrhea in infants.

Keywords: diarrhea in children, diarrhea risk, foods, water sanitation, breastfeeding, Factors determinants.

\section{INTRODUCTION}

According to the World Health Organization pThe definition of diarrhea is defecation with a liquid consistency (loose stools) 3 or more times a day (24 hours). There are two important criteria that must be remembered, namely liquid and frequent bowel movements, if you have three bowel movements a day but are not liquid, then it cannot be called daire. Or if you have runny stools but not three times a day, then it is not diarrhea (Betz, 2009).Diarrhea is the number 2 cause of death in children under five in the world, number 3 in infants, and number 5 for all ages. Globally each year there are about 2 billion cases of diarrhea with a death rate of 1.5 million per year. In developing countries, children under 3 have an average of 3 episodes of diarrhea per year. Each episode of diarrhea will cause a loss of nutrients needed by children to grow, so that diarrhea is the main cause of malnutrition in children (WHO, 2009). 
In Indonesia, diarrhea is the main cause of death for infants and children under five (children aged 1 month to $<5$ years). Riskesdas, (2013) has collected information on the incidence of diarrhea so that the program can use it, and the prevalence period of diarrhea so that it can be compared with Riskesdas 2007. Based on the results of basic health research (Riskesdas, 2007) conducted by the Ministry of Health cq the National Research and Development Agency in 2007, diarrhea disease became the main cause of death for infants $(31.4 \%)$ and children under five $(25.2 \%)$. Meanwhile, the prevalence period of diarrhea in Riskesdas 2013 was found (3.5\%) to be smaller than Riskesdas 2007 (9.0\%). The incidence of diarrhea in children aged 0-11 months (7.0\%) and children aged 12-23 months (9.7\%). In DI Yogyakarta, the incidence of diarrhea among children under five (5.0\%). (Riskesdas, 2013).

In rural Mozambique, factors that can influence the occurrence of diarrhea in children aged 0-11 months and children aged 24-59 months, one of which is the source of water or depending on the quality of water used for daily activities (Nhampossa $T$, et al, 2015). Meanwhile according to (Agustina R, et al, 2013)the risk of experiencing diarrhea increases in children aged $<2$ years, namely from food factors. This can be found also in research conducted by a number of developing countries such as Vietnam, Thailand, and Bangladesh, that some mothers have poor food-hygiene practices which can make children $<2$ years old more susceptible to diarrhea. This finding is also in line with the previous review that diarrheal disease is very high during the weaning period (6-24 months). According to (Conkle J, et al, 2016) researchers found evidence that diarrhea in children aged 10 months. Breastfeeding was associated with lower diarrhea outcome $(\mathrm{OR}=0.59,95 \%$ CI 0.43-0.82). Compared with those who were given consumption of candy and consumption of milk, there was an increase in the prevalence of diarrhea $(\mathrm{OR}=1.64,95 \% \mathrm{CI} 1.22-2.22$ and $\mathrm{OR}=1.67,95 \% \mathrm{CI} 1.21-2.30)$.

From several reviews that have been found that factors that can affect the incidence of diarrhea in infants include breastfeeding, food factors, and water sources. The purpose of this review is the importance for families to maintain breastfeeding for 2 full years, pay attention to water sources used for daily purposes, especially water for drinking, the importance of choosing good and appropriate food for children. This review aims to summarize and synthesize (breastfeeding, food and water sources).

\section{Formulation of the problem:}

What factors can affect the incidence of diarrhea in infants aged 0 to 2 years (breastfeeding, food and water sources).

Aim:

Analyzing the factors that influence the incidence of diarrhea in infants aged 0-2 years.

\section{RESEARCH METHODS}

The type of research used is literature review. Literature review research is a comprehensive study and interpretation of literature related to a particular topic that begins by determining research questions and then answering them by searching and analyzing appropriate literature, using a systematic approach. Literature review is an important way because it provides a research synthesis of information on a particular topic (Aveyard, 2010).

Sources of information: A comprehensive literature search was performed using the Science Direct, Pubmed, and Google Schoolar databases. Literature searches are limited to those published in 2009 to 2018 . 
The steps taken in this review consisted of: (I) identifying research questions; (II) identify relevant studies; (III) article selection; (IV) charting data; and (V) compile, summarize and report the results.

Stage I. Identifying research questions.

The aim of this review article is to identify and determine the nature and level of literature on factors that influence the incidence of diarrhea in infants aged 0-2 years.

Stage II. Identify relevant studies

a Search strategy: a comprehensive search strategy was carried out separately using the Medical Subject Heading (MeSH) Boolean method (AND, OR, NOT) of both terms and free text keywords at the initial search stage. Next, look for results and combine them to identify relevant articles. The first search is diarrhea in infants, then the second search is the term factors that affect the incidence of diarrhea as shown in table 1, then the two terms are combined to get the final article.

Table 1. Documentation of keywords used for literature searches

\begin{tabular}{|c|l|l|}
\hline Code & \multicolumn{1}{|c|}{ Medical Subject Heading (MeSH) } & \multicolumn{1}{c|}{ Keyword } \\
\hline \#A & Diarrhea in children, diarrhea risk, & $\begin{array}{l}\text { "Diarrhea in children" "diarrhea risk" OR "foods" } \\
\text { OR "feeding" }\end{array}$ \\
\hline \#B & Factors, determinants & Factors OR determinats \\
\hline \#C & $\begin{array}{l}\text { ("Diarrhea in children" AND ("diarrhea and foods" OR ("diarrhea" AND "risk") OR "foods" OR } \\
\text { "diarrhea and foods" AND "water sanitation" OR "diarrhea and water drinking" AND } \\
\text { "breastfeeding" " diarrhea and breastfeeding "(determinants OR factors)) }\end{array}$ \\
\hline
\end{tabular}

Stage III. Article selection

Review and filter the data that has been obtained. During the identification search, there were 60 articles from the total database used. After duplicate removal and screening of titles for relevance. Further screening of titles and abstracts obtained 10 journals for review. Figure 1 provides a chart of the literature search process.

Stage IV. charting data

Furthermore, the 10 literatures will be analyzed using the main predetermined main criteria such as study location, study population, objectives / objectives of the study, methodology, whether it is significant or not, as well as findings / recommendations (attached to attachment 1) then comparing the data taken.

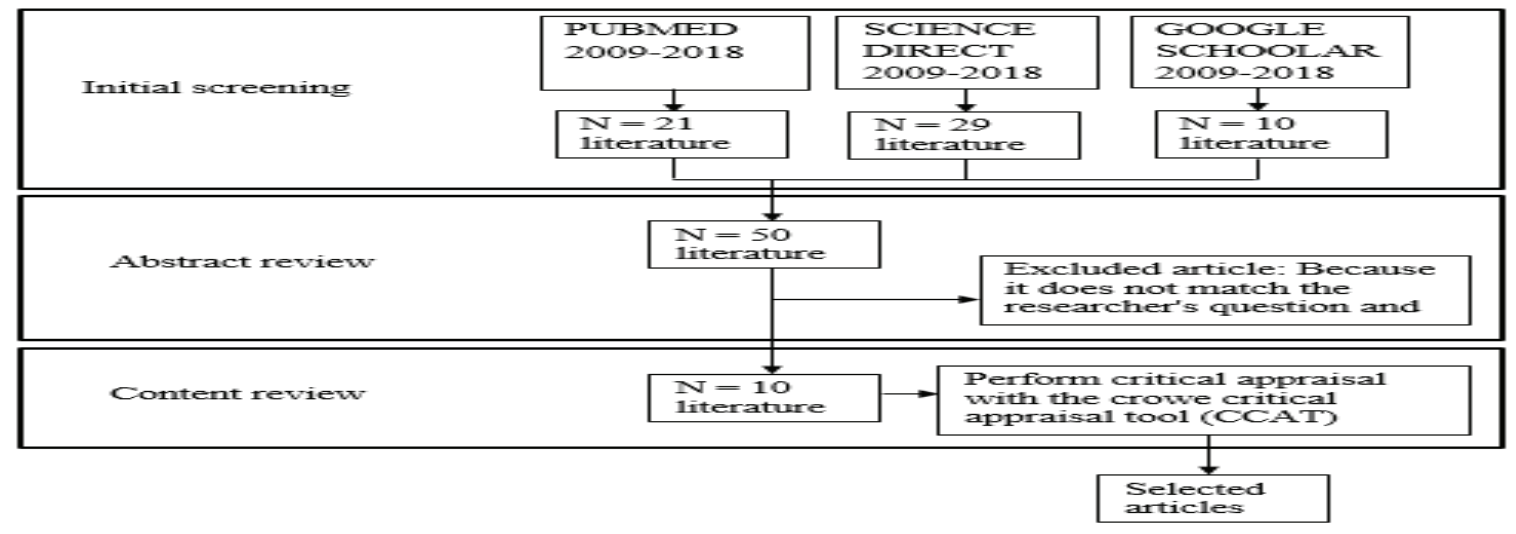

Figure 1.Selected Search Results and Literature

\section{ANALYSIS RESULTS}


1. According to research conducted by Joel Conkle, Usha Ramakrishnan, and Matthew C. Freeman (2016). With the title "Prechewing infant food, consumption of sweets and dairy and not breastfeeding are associated with increased diarrhea risk of 10-month-old infants in the United States". Just as expected, infants 10 months of age who were breastfed or who were fully breastfed when associated with diarrhea appeared to have lower scores $(\mathrm{OR}=$ $0.59,95 \%$ CI $0.43-0.82$ ), while children who received food and sweets or milk was associated with an increase in the prevalence of diarrhea, namely (OR = $1.64,95 \%$ CI $1.22-2.22$ and $\mathrm{OR}=1.67,95 \%$ CI 1.21-2.30). In the adjusted model, breastfeeding remains a statistically significant protective feeding practice, whereas candy and milk consumption have been shown to increase the risk of diarrhea. Thus the highest rate of diarrhea was found among children who received food and candy or milk.

2. According to Zivich P, et all, (2018). With the title "Implementation of Steps 19 to Successful Breastfeeding Reduces the Frequency of Mild and Severe Episodes of Diarrhea and Respiratory Tract Infection Among 0-6 Month Infants in Democratic Republic of Congo". From the results of his research, it has found evidence that breastfeeding or breastfeeding in the first 6 months can provide relief to children who are suffering from diarrhea. Optimal breastfeeding is known to protect against diseases, one of which is diarrhea. The incidence of diarrhea was lower among infants who received the form of breastfeeding support provided by health care workers during infant clinic visits and educational materials compared with the control group (IRR $0.72 ; 95 \%$ CI 0.53 , 0.99). And hospitalization (IRR $0.42 ; 95 \%$ CI 0.17, 1.06), the application of "bypass" of the Ten Steps to Successful Breastfeeding have been found to reduce diarrhea. When compared with the group of mothers and babies who do not apply breastfeeding. Although not statistically significant, the incidence rate of diarrhea was higher among infants receiving care at the clinic implementing the 1-10 steps group (IRR 1.24; 95\% CI 0.93, 1.68), compared to the control clinic. However, the steps 1-10 groups had a lower rate of diarrhea episodes resulting in hospitalization (IRR 0.12; 95\% CI 0.03, 0.53). however, the incidence rate of diarrhea was higher among infants who received care at the clinic implementing the step 1-10 group (IRR 1.24; 95\% CI 0.93, 1.68), compared to the control clinic. However, the steps 1-10 group had a lower rate of diarrhea episodes resulting in hospitalization (IRR $0.12 ; 95 \%$ CI 0.03, 0.53). however, the incidence rate of diarrhea was higher among infants who received care at the clinic implementing the step $1-10$ group (IRR 1.24; 95\% CI 0.93, 1.68), compared to the control clinic. However, the steps 1-10 groups had a lower rate of diarrhea episodes resulting in hospitalization (IRR $0.12 ; 95 \%$ CI $0.03,0.53$ ).

3. According to research by Lea A. Cupul-Uicaba, et al, (2017). With the title "In utero exposure to DDT and incidence of diarrhea among boys from tropical Mexic". As we know that exclusive breastfeeding is breast milk that is given to babies from 0 to 6 months of age but in most cases compared to urban settings, children from rural areas are more likely because one of the reasons is that many children in rural areas start to drink milk other than breast milk. after four months of age (48.8\% vs $36.6 \%)$. A slightly higher percentage of children from rural areas had at least one episode of diarrhea (83.3\%) compared to those from urban areas $(81.5 \%)$. Overall, the incidence (IR) rate of diarrhea was 1.7 per 
child year; IR was slightly higher in rural children (1, 81 per year of children) were compared with children from urban (1.62 per child) settings (unadjusted incidence rate of nonadjustment, $\mathrm{p}<0.01$ ). Even so, at least children from rural areas had an average duration of breastfeeding every 3 months longer than those from urban areas. Breastfeeding reduces the risk of gastrointestinal infections by up to $40 \%$ in the first year of life (Kramer et al., 2001; Oddy, 2001). Selective colonization of the gut microbiota due to breastfeeding appears to aid the development of the infant's immune system (Abrahamsson et al., 2015; Backhed et al., 2015; Ottman et al., 2012). But the duration of breastfeeding did not modify the association with DDE in this study. Even so, at least children from rural areas had an average duration of breastfeeding every 3 months longer than those from urban areas. Breastfeeding reduces the risk of gastrointestinal infections by up to $40 \%$ in the first year of life (Kramer et al., 2001; Oddy, 2001). Selective colonization of the gut microbiota due to breastfeeding appears to aid the development of the infant's immune system (Abrahamsson et al., 2015; Backhed et al., 2015; Ottman et al., 2012). But the duration of breastfeeding did not modify the association with DDE in this study. Even so, at least children from rural areas had an average duration of breastfeeding every 3 months longer than those from urban areas. Breastfeeding reduces the risk of gastrointestinal infections by up to $40 \%$ in the first year of life (Kramer et al., 2001; Oddy, 2001). Selective colonization of the gut microbiota due to breastfeeding appears to aid the development of the infant's immune system (Abrahamsson et al., 2015; Backhed et al., 2015; Ottman et al., 2012). But the duration of breastfeeding did not modify the association with DDE in this study. Selective colonization of the gut microbiota due to breastfeeding appears to aid the development of the infant's immune system (Abrahamsson et al., 2015; Backhed et al., 2015; Ottman et al., 2012). But the duration of breastfeeding did not modify the association with DDE in this study. Selective colonization of the gut microbiota due to breastfeeding appears to aid the development of the infant's immune system (Abrahamsson et al., 2015; Backhed et al., 2015; Ottman et al., 2012). But the duration of breastfeeding did not modify the association with DDE in this study.

4. According to Nhampossa T, et al, (2015). With the title "Diarrheal Disease in Rural Mozambique: Burden, Risk Factors and Etiology of Diarrheal Disease among Children Aged 0-59 Months Seeking Care at Health Facilities". One of the factors that can influence the occurrence of diarrhea in children aged 0-11 months and children aged 24-59 months, one of which is water sources or depending on the quality of water used for daily purposes. Some people used tap water (36\% for cases vs $31 \%$ for controls) and drill water ( $25 \%$ for cases vs $24 \%$ for controls). The enormous wealth of households ( $83 \%$ for cases vs $83 \%$ for controls) of having better access to water does not appear to have an effect on the risk of diarrhea. But treating chlorinated water or water was associated with an increased risk of diarrhea in children aged $0-11$ months (OR 1.93; 95\% IC 1, $27-2.92, p=0.002)$. And giving children stored water was also associated with an increased risk of MSD in children aged 0-11 months $(\mathrm{p}<0.001)$.

5. Likewise with the research conducted (Larson L, et al, 2016) with the title "Acceptance and Impact of Point-of-Use Water Filtration Systems in Rural Guatemala ". The use of clean water in daily needs is very influential on health, 
especially when water is used for drinking. So the researchers are looking for a truth about the effect of diarrhea and the type of water used, the results obtained are that from 40 households with water processed through filters, there were six cases of diarrhea $(15 \%)$, while in 16 households with damaged water filters, five cases of diarrhea (31\%). Of the 34 households with concrete or wooden floors, five cases of diarrhea $(14.7 \%)$, while in 22-holding houses with dirt floors, six cases of diarrhea $(27.2 \%)$ were reported (Table 3$)$. Although not statistically significant due to the small sample size, the likelihood of reported diarrhea cases was 2.15 times higher in houses with dirt floors $(\mathrm{OR}=2.15,95 \%$ confidence interval [CI] 0.6, 8,

6. In another study conducted by Bhavnani D, et all, (2018). With the title "Impact of Rainfall on Diarrheal Disease Risk Associated with Unimproved Water and Sanitation". The incidence of diarrhea caused by water sources, after adjusting for children under 5 years, poor water sources or poor sanitation, poor water sources and improper sanitation were associated with increased diarrhea $(\mathrm{OR}=$ $3.6,95 \% \mathrm{CI}=1.7-7.8$ and $\mathrm{OR}=1.7,95 \% \mathrm{CI}=1.2-2.5$, respectively). Effect modification by water and sanitation. Water sources and sanitation significantly modified their relationship with diarrhea. It predicted that diarrhea associated with insignificant water sources was only statistically significant in households with improved sanitation $(\mathrm{OR}=7.1,95 \% \mathrm{CI}=2.4-21.3)$. As well,

7. According to Kyle S. Enger, et al, (2012). With the title "The joint effects of efficacy and compliance: A study of household water treatment effectiveness against childhood diarrhea". Diarrhea in children can be caused by water availability and drinking water consumption habits. In the research results of Kyle S. Enger, et al. Find evidence that the risk of diarrhea, by applying selfhabits to household water treatment is a very large benefit, where $80 \%$ of children consume treated water $100 \%$ of the time, and $20 \%$ of children consume only untreated water. The benefits are smaller, where $40 \%$ of children consume treated water $100 \%$ of the time, $50 \%$ of children consume treated water $80 \%$ of the time, and $10 \%$ of children consume only untreated water.

8. Meanwhile, according to research by George P. Einstein, et al, (2017). With the title "Meta-analysis of staphylococcal diarrhea in some developing African countries". Children who had an inadequate sanitary condition were five times more likely $(\mathrm{OR}=5.307, \mathrm{P}=0.026,95 \% \mathrm{CI}: 2.878,9.613)$ to have a staphylococcal diarrhea condition than those who had an adequate sanitary condition. Also, children who were not exposed to food prior to exclusive breastfeeding were twice as likely $(\mathrm{OR}=2,214, \mathrm{P}=0.033,95 \% \mathrm{CI}: 1.774$, 4.683 ) to develop the condition of staphylo-coccal diarrhea compared with those exposed to foods given before 6 months of age. It is quite clear that sanitation conditions and the achievement of exclusive breastfeeding can have an influence on the incidence of diarrhea in children, especially in children less than 12 months, which is the highest reported positive for diarrhea (20.8\%). Meanwhile children between 13 and 24 months reported the least positive (3.9) among the subjects.

The results of the study found that less than a quarter $(21.9 \%)$ were exposed to adequate sanitation conditions while more than half $(78.1 \%)$ were exposed to poor and inadequate sanitary conditions. In addition, more than one third of 
them $(38.9 \%)$ children who were exclusively breastfed by their mother, namely $61.1 \%$ did not get diarrhea.

9. Factors that cause diarrhea in children can be caused by bacteria and viruses, poor water sanitation, not achieving exclusive breastfeeding, even food. As in the research of Avita A. Usfar, DrScHum, et al, (2010). With the title "Food and Personal Hygiene Perceptions and Practices among Caregivers Whose Children Have Diarrhea: A Qualitative Study of Urban Mothers in Tangerang, Indonesia". Which discusses the relationship between food factors and the incidence of diarrhea in children. The results of his research suggest that diarrhea that occurs in children is caused by not paying attention to food hygiene such as choosing food ingredients, washing food ingredients before cooking, closing food, or the habit of buying ready-to-eat food.

According to Avita A. Usfar, DrScHum, et al, (2010). Concept, Causes, and Transmission of Diarrhea as Attended by Mother. Most of the mothers $(n=15$; $63 \%$ ) admitted that their children had diarrhea based on stool consistency (liquid stool), while a few others $(\mathrm{n}=9 ; 38 \%)$ identified diarrhea by combining stool consistency and frequency (more than 3\%). times a day). Foodstuffs believed to cause diarrhea include legumes, ice, spicy and sour foods, candy, snacks, and coffee (total $n 1 / 413 ; 50 \%)$. Some mothers $(n=9 ; 38 \%)$ believed that the causes of diarrhea were cold, exposure to dirty environments, or improper food. There were also most of the mothers who assumed that $(n=19 ; 80 \%)$, considered that diarrhea was also related to physical development and motor development of children, such as crawling, walking, growing, talking, and teething. The concept of transmission of diarrhea through unclean hands or child-to-child transmission was recognized by 9 mothers $(38 \%)$.

Most of the children $(\mathrm{n}=13 ; 54 \%)$ in the study had already consumed family food, while the other $10(42 \%)$ still consumed rice porridge. To save time and work, children's meals are usually prepared at the same time as the mother preparing family meals. This practice was consistently discovered during interviews and observation. Children were given food when it was hot or warm (within 15 minutes after cooking) (n $1 / 4$ 12;50\%) or when cold (within 30 minutes after cooking) (n $1 / 411 ; 46 \%)$.

10. Meanwhile, according to Strina A, et al, (2012). With the title "Factors associated with rotavirus diarrhoea in children living in a socially diverse urban center in Brazil". One of the factors for the occurrence of diarrhea in children aged $<12$ months, and / or aged 12-23 months, and / or aged 24-59 months, and the remaining 60-120 months, is caused by food and the success of breastfeeding. The study found evidence that current breastfeeding deficiency was significantly associated with a 149\% higher chance of developing diarrhea. Lack of breastfeeding has high individual PAF (17\%). While risk factors for food preparation include children buying or receiving food in non-domestic spaces, mothers or regular food providers who do not pay attention to the temporary food consumed and children who drink potentially contaminated water. Or the child consumes food outside the home (PAF 7\%). So we can conclude that exclusive breastfeeding can reduce diarrhea disease in children. The more often the child breastfeeds the immunity in the body the better. Likewise, choosing food or giving food according to age will provide opportunities for healing or even prevent diarrhea in children. 


\section{RESULTS AND DISCUSSION}

HTA analysis in the use of a form of breastfeeding support provided by health care workers during infant clinic visits and educational materials, this is very good to be applied in all facilities or clinics in order to improve the quality of breastfeeding or exclusive breastfeeding programs for 6 months. Given that it is still difficult to familiarize people with always giving exclusive breastfeeding to their children, the implementation of "shortcuts" from the Ten Steps to Successful Breastfeeding is quite helpful in overcoming the incidence of diarrhea in infants, especially those aged 0 to 2 years. In the research of Zivich P, et all, (2018). Has found an extraordinary outcome compared to the control group (IRR 0.72; 95\% CI 0.53, 0.99). And hospitalization (IRR $0.42 ; 95 \%$ CI 0.17, 1.06), the application of "bypass" of the Ten Steps to Successful Breastfeeding have been found to reduce diarrhea. When compared with the group of mothers and babies who do not apply breastfeeding. Although not statistically significant, the incidence rate of diarrhea was higher among infants receiving care at the clinic implementing the $1-10$ steps group (IRR $1.24 ; 95 \%$ CI 0.93 , 1.68), compared to the control clinic. The use of water sources for both hygiene and drinking, the use of water filters is very helpful in reducing diarrhea in families, especially children, as in the study however, the incidence rate of diarrhea was higher among infants who received care at the clinic implementing the step 1-10 group (IRR 1.24; $95 \%$ CI 0.93 , 1.68), compared to the control clinic. The use of water sources for both hygiene and drinking, the use of water filters is very helpful in reducing diarrhea in families, especially children, as in the study however, the incidence rate of diarrhea was higher among infants who received care in the clinic implementing the step 1-10 group (IRR $1.24 ; 95 \%$ CI $0.93,1.68)$, compared to the control clinic. The use of water sources for both hygiene and drinking, the use of water filters is very helpful in reducing diarrhea in families, especially children, as in the study(Larson L, et al, 2016). Seventy-nine percent $(n=56)$ of 71 households that received water filters in 2014 participated in this study. The majority of the families $(71.4 \% ; \mathrm{n}=40)$ used water filters and 16 families (28.6\%) had damaged water filters. Of the families with working water filters, $15 \%$ reported diarrhea, while $31 \%$ of families with damaged water filters reported diarrhea. Only $55.4 \%$ of the houses have concrete floors. More households with dirt floors and bro-ken water filters are reporting cases of diarrhea at this time. A review of records of aten-dees in outreach clinics noted a decrease in intestinal infections from 2014 (53\%) to 2015 (32\%). As we know, food can also have a big influence on the incidence of diarrhea in infants, in research conductedAvita A. Usfar, DrScHum, et al, (2010). Based on what has been studied regarding the concept, causes, and transmission of diarrhea as noted by mothers. The results of his research suggest that diarrhea that occurs in children is caused by not paying attention to food hygiene such as choosing food ingredients, washing food ingredients before cooking, closing food, or the habit of buying ready-to-eat food. This can be proven from several statements of mothers who assume or may be said to have experienced it. Most of the mothers $(n=15 ; 63 \%)$ admitted that their children had diarrhea based on stool consistency (liquid stool), while a few others $(\mathrm{n}=9 ; 38 \%)$ identified diarrhea by combining stool consistency and frequency (more than 3\%). times a day). Foodstuffs believed to cause diarrhea include peas, ice, spicy and sour foods, candy, snacks, and coffee (total n $1 / 413 ; 50 \%$ ). Some women $(\mathrm{n}=9 ; 38 \%)$ believed that the cause of diarrhea was cold, exposure to dirty 
environment, or improper food. There were also most mothers who assumed that $(\mathrm{n}=$ $19 ; 80 \%$ ), considered that diarrhea was also related to physical development and motor development of children, such as crawling, walking, growing, talking, and teething. The concept of transmission of diarrhea through unclean hands or child-to-child transmission was recognized by 9 mothers $(38 \%)$. consider that diarrhea is also related to physical development and motor development of children, such as crawling, walking, growing, talking, and teething. The concept of transmission of diarrhea through unclean hands or child-to-child transmission was recognized by 9 mothers $(38 \%)$. consider that diarrhea is also related to physical development and motor development of children, such as crawling, walking, growing, talking, and teething. The concept of transmission of diarrhea through unclean hands or child-to-child transmission was recognized by 9 mothers $(38 \%)$.

\section{CONCLUSION}

According to several researchers who have been discussed in the results chapter, diarrhea incidence factors such as breastfeeding, food, and water sources can have a significant influence on diarrhea, especially in children aged 0-2 years or under 5 years of age. According to the literature, there are several studies conducted by Joel Conkle, Usha Ramakrishnan, and Matthew C. Freeman (2016)., Nhampossa T, et al, (2015)., Larson L, et al, (2016)., Zivich P, et al. all, (2018)., Bhavnani D, et all, (2018)., Lea A. Cupul-Uicaba, et al, (2017)., George P. Einstein, et al, (2017)., Avita A. Usfar , DrScHum, et al, (2010)., Strina A, et al, (2012)., And Kyle S. Enger, et al, (2012). Exclusive breastfeeding for up to 6 months can reduce the risk of diarrhea in babies, daily use of water comes from clean water sources.

\section{REFERENCES}

Agostino Strina, et al, 2012. Factors associated with rotavirus diarrhea in children living in a socially diverse urban center in Brazil. Transactions of the Royal Society of Tropical Medicine and Hygiene: Brazil.

Avita A. Usfar, DrScHum, et al 2010. Food and Personal Hygiene Perceptions and Practices among Caregivers Whose Children Have Diarrhea: A Qualitative Study of Urban Mothers in Tangerang, Indonesia. Journal of Nutrition Education and Behavior: Indonesia.

Betz Cecily L, Sowden Linda A. 2009. Nursing pocket book. Pediatik, Jakarta, EGC.

Darlene Bhavnani, et al, 2014. Impact of Rainfall on Diarrheal Disease Risk Associated with Unimproved Water and Sanitation. The American Society of Tropical Medicine and Hygiene.

George P. Einstein, Orien L. Tulp, Temitope C. Oyesile, Olayide F. Obidi 2017. Metaanalysis of staphylococcal diarrhea in some developing African countries. BeniSuef University Journal of Basic and Applied Sciences: USA

Joel Conkle, Usha Ramakrishnan and Matthew C. Freeman, 2017. Prechewing infant food, consumption of sweets and dairy and not breastfeeding are associated with increased diarrhea risk of 10-month-old infants in the United States. Maternal and child nutrition: Georgia.

Kyle S. Enger, et al, 2012. The joint effects of efficacy and compliance: A study of household water treatment effectiveness against childhood diarrhea. Sciverse Science Direct: USA. 
Kim L. Larson, PhD, MPH, RN, Corrie Hansen, BSN, RN, Michala Ritz, MPH, BS, \& Diego Carre no, 2016. Acceptance and Impact of Point-of-Use Water Filtration Systems in Rural Guatemala. NursingScholarship: USA

Lea A. Cupul-Uicaba, b, Efraín A. Terrazas-Medinaa, Mauricio Hernández-Ávilaa, Matthew P. Longneckerc 2017.In utero exposure to DDT and incidence of diarrhea among boys from tropical Mexic. Environmental Research: USA

Paul Zivich1, Bruno Lapika, Frieda Behets, Marcel Yotebieng, 2018. Implementation of Steps 1-9 to Successful Breastfeeding Reduces the Frequency of Mild and Severe Episodes of Diarrhea and Respiratory Tract Infection Among 0-6 Month Infants in Democratic Republic of Congo. Maternal and Child Health Journal: USA.

Tacilta Nhampossa, et al, 2014. Diarrheal Disease in Rural Mozambique: Burden, Risk Factors and Etiology of Diarrheal Disease among Children Aged 0-59 Months Seeking Care at Health Facilities. Plos one: USA.

World Health Organization. 2009. World Health Statistics.

Yogyakarta City Health Office. 2015. Yogyakarta City Health Profile 2015 (Data Year 2014). Yogyakarta City Government Health Office: Yogyakarta. 